\title{
Assessment of inflammatory parameters in obstructive coronary artery disease and cardiac syndrome $X$ : an evolving value of neutrophil-lymphocyte ratio
}

\author{
Bedrettin Boyraz $^{1} \odot$, Ferit Onur Mutluer ${ }^{2} \odot$, Dursun Topal ${ }^{3} \odot$, Mehmet Demir $^{3} \odot$, Tezcan Peker $^{4} \odot$, \\ Mustafa Yılmaz ${ }^{5}$, Alkame Akgümüş ${ }^{6} \odot$, Erhan Tenekecioğlu ${ }^{3} \odot$
}

${ }^{1}$ Department of Cardiology, Tatvan State Hospital, Bitlis, Turkey

${ }^{2}$ Department of Cardiology, Koç University School of Medicine, Istanbul, Turkey

${ }^{3}$ Department of Cardiology, University of Health Sciences, Bursa Yüksek İhtisas Training and Research Hospital, Bursa, Turkey

${ }^{4}$ Department of Cardiology, Bursa Doruk Hospital, Bursa, Turkey

${ }^{5}$ Department of Cardiology, Uludağ University School of Medicine, Bursa, Turkey

${ }^{6}$ Department of Cardiology, Gemlik State Hospital, Bursa, Turkey

\section{ABSTRACT}

Objectives: Atherosclerosis represents an active inflammation that leukocytes play a major role. Neutrophillymphocyte ratio (NLR) has been shown as an indicator of systemic inflammation. Our aim was to evaluate inflammatory markers in obstructive coronary artery disease (CAD) and cardiac syndrome X (CSX) and to evaluate NLR in predicting CAD in patients with typical chest pain and coronary risk factors.

Methods: Eighty patients with CSX, 80 patients with obstructive CAD with unstable angina pectoris and a control group of 80 subjects were recruited into the study. Hematologic and biochemical parameters were investigated.

Results: High-sensitive C-reactive protein (hs-CRP) was increased in CAD group and CSX group compared to the control group $(p<0.001)$; however it was comparable between CAD and CSX groups $(p=0.065)$. Mean NLR was higher in CAD group than CSX group and control group that the lowest value was in the control group. In CAD group, hs-CRP was positively correlated with NLR $(\mathrm{r}=0.43, p<0.001)$, and Gensini score ( $\mathrm{r}$ $=0.54, p<0.001)$. NLR was also linearly correlated with Gensini score $(\mathrm{r}=0.49, p<0.001)$. In ROC curve analysis, for the NLR of 2.26 , NLR values attained $85.5 \%$ sensitivity and $43.9 \%$ specificity $(p<0.001)$. When cut-off value was taken as $3.05 \mathrm{mg} / \mathrm{dL}$ for CRP, CRP values attained $78.7 \%$ sensitivity and $43.6 \%$ specificity $(p<0.001)$. When cut-off value was taken as $9.5(\times 109 / \mathrm{L})$ for white blood cell (WBC), WBC values attained $58.8 \%$ sensitivity and $34.3 \%$ specificity $(p<0.001)$.

Conclusions: NLR seems to be usefull in prediction of obstructive CAD and can be implemented in planning for diagnostic procedures in patients with typical chest pain. Increased CRP, WBC and NLR may give insight about main pathology of CSX. Despite absence of obstructive narrowing in epicardial coronaries, it appears be salutary to treat CSX due to the similar underlying pathophysiology with obstructive CAD.

Keywords: obstructive coronary artery disease, cardiac syndrome X, neutrophil-lymphocyte ratio 
$\Delta$ inv therosclerosis represents an active inflammation in which leukocytes play a major role during the atheromatous plaque formation and prograssion [1] The subtypes of white blood cells have been investigated for their roles in atherosclerosis intensively [2]. Mononuclear cells (monocytes, macrophages, T-lymphocytes) have indispensable roles within the atherosclerotic plaques [3]. Neutrophils have functions in leukocyte-platelet aggregate formation and reperfusion injury but also are involved in myocardial infarct healing. Neutrophils and lymphocytes indicate opposing clinical outcomes in acute coronary syndromes (ACS) [4]. The neutrophil-lymphocyte ratio (NLR) was reported as an independent predictor for mortality and myocardial infarction in coronary artery disease [5]. NLR has also been reported as a prognostic marker in non-cardiac disease and in patients undergoing percutaneous coronary intervention. Within the markers of inflammation in coronary artery disease NLR has been reported the greatest predictive power for poor outcomes in patients with coronary artery disease [6-8].

Patients having typical chest pain associated with ischaemic ST-segment changes on exercise stress testing or ischaemic findings at myocardial perfusion scintigraphy and with normal epicardial coronaries in coronary angiography are diagnosed as cardiac syndrome X (CSX) [9]. The prominent mechanism is vascular endothelial dysfunction associated with microvascular inflammation [10].

The aim of the current study was to evaluate the inflammatory, biochemical and haematologial parameters in patients with obstructive coronary artery disease (CAD) and CSX and to investigate the predictive role of NLR for CAD and CSX.

\section{METHODS}

This study was designed as a cross-sectional and observational study. For this study, totally 630 subjects were reviewed in detail. Eighty patients diagnosed with CSX who were admitted to our hospital, 80 patients with obstructive CAD with unstable angina pectoris and a control group of 80 subjects were recruited into the study between April 2012 and November 2013, after the approval of local ethics committee. CSX was defined as typical chest pain during rest or effort, abnormal test result for exercise electrocardiography (ECG) and myocardial perfusion scintigraphy and the presence of angiographically normal epicardial coronary arteries. Control group was selected from volunteers presented to our hospital with the complaint of atypical angina who had similar risk profile with the patients (diabetes [DM], hypertension [HT], age, gender) and had normal coronaries at coronary angiography. Patients with valvular heart disease (mitral stenosis and moderate to severe mitral regurgitation, moderate to severe aortic stenosis and regurgitation including mitral annular calcification), heart failure (left ventricular ejection fraction [LVEF] $<50 \%$ ), congenital heart disease, cardiomyopathy, left ventricular hypertrophy, right ventricular hypertrophy, cardiac surgery, thyroid disease, anemia, infectious or pulmonary diseases were not included into the control group and patient group with CSX. Patients with CAD had single vessel or multivessel disease, according to the number of epicardial arteries with at least one lesion measuring $\geq 50 \%$ diameter stenosis and typical angina pectoris in whom cardiac necrosis markers were not elevated.

blood is collected in a tube containing K3 EDTA for measurement of hematologic indices in all patients undergoing the coronary angiography. Hematologic indices are evaluated from complete blood count (CBC) analysis performed by a Coulter LH 780 Hematology Analyzer (Beckman Coulter Ireland Inc. Mervue, Galway, Ireland). Lipid serum levels were measured immediately by routine methods. Serum total triglyceride (TG) and total cholesterol (TC) were measured by enzymatic methods on an automatic biochemical analyzer. Serum high-density lipoproteincholesterol (HDL-C) was tested by magnesium tungstate phosphate sedimentation and the concentration of low-density lipoprotein-cholesterol (LDL-C) was calculated by the Friedewald formula as follows: LDL-C $(\mathrm{mmol} / \mathrm{L})=\mathrm{TC}-\mathrm{HDL}-\mathrm{C}-\mathrm{TG} / 2.2$ (TG $<4.5 \mathrm{mmol} / \mathrm{L})$. Fasting blood samples were also collected in tubes containing citrate, and were drawn and centrifuged immediately. Serum was then aliquoted and stored at $-80^{\circ} \mathrm{C}$ until analysis for Creactive protein (CRP) measurement. No specimen inadvertently thawed during storage. CRP measurements were performed on the COBAS Integra (Roche Diagnosis Limited, East Sussex, United 
Table 1. Clinical characteristics of study groups

\begin{tabular}{|c|c|c|c|}
\hline Groups & $\begin{array}{l}\text { Control } \\
(\mathrm{n}=\mathbf{8 0})\end{array}$ & $\begin{array}{c}\text { CSX } \\
(n=80)\end{array}$ & $\begin{array}{c}\text { CAD } \\
(n=80)\end{array}$ \\
\hline Males/Females & $40 / 40$ & $43 / 37$ & $47 / 33$ \\
\hline Age (years) & $47.9 \pm 6$ & $49.2 \pm 10$ & $61.2 \pm 9^{*}$ \\
\hline $\operatorname{BSA}\left(\mathrm{m}^{2}\right)$ & $1.78 \pm 0.14$ & $1.76 \pm 0.16$ & $1.77 \pm 0.12$ \\
\hline BMI $\left(\mathrm{kg} / \mathrm{m}^{2}\right)$ & $26.5 \pm 2.9$ & $27.6 \pm 3.5^{\dagger}$ & $27.8 \pm 2.4$ \\
\hline Systolic blood pressure $(\mathrm{mmHg})$ & $120.1 \pm 10.7$ & $123.6 \pm 9.3$ & $122.6 \pm 13.6$ \\
\hline Diastolic blood pressure $(\mathrm{mmHg})$ & $74.2 \pm 7,4$ & $73.6 \pm 5,2$ & $74.2 \pm 9$ \\
\hline Heart rate (bpm) & $76 \pm 8$ & $74 \pm 10$ & $81 \pm 13^{\dagger}$ \\
\hline Smoking, n (\%) & $28(35 \%)$ & $30(37 \%)$ & $33(41 \%)$ \\
\hline Hypertension, n (\%) & $33(41 \%)$ & $34(42 \%)$ & $36(45 \%)$ \\
\hline Diabetes mellitus, n (\%) & $25(31 \%)$ & $20(25 \%)$ & $25(31 \%)$ \\
\hline Hyperlipidemia, n (\%) & $26(32 \%)^{*}$ & $22(28 \%)$ & $52(65 \%)^{* \pi}$ \\
\hline $\operatorname{LVEF}(\%)$ & $61.1 \pm 3.0$ & $60.4 \pm 2.8$ & $47.3 \pm 9.7^{* \pi}$ \\
\hline Gensini score & - & - & $21.45 \pm 15.49$ \\
\hline
\end{tabular}

Data are shown as mean \pm standard deviation or number (percent). BMI $=$ body mass index, BSA $=$ body surface area, $\mathrm{CAD}=$ coronary artery disease, $\mathrm{CSX}=$ cardiac syndrome $\mathrm{X}, \mathrm{LVEF}=$ left ventricular ejection fraction, ${ }^{*} p<$ 0.001 versus control group, ${ }^{\uparrow} p<0.001$ versus $\operatorname{CSX}$ group, ${ }^{\dagger} p<0.05$ versus control group

Kingdom) using the CRP-latex assay in both the highsensitivity application (analytical range, 0.2 to 12 $\mathrm{mg} / \mathrm{l}$ ) and the normal application (analytical range, 2 to $160 \mathrm{mg} / \mathrm{l})$.

\section{Statistical Analysis}

Body mass index (BMI) was calculated by the common formula: BMI $(\mathrm{kg} / \mathrm{m} 2)=$ weight $(\mathrm{kg}) /$ height (m2). Body surface area (BSA) was assessed by a variation of the DuBois and DuBois formula: BSA $(\mathrm{m} 2)=$ [weight $(\mathrm{kg}) 0.425 \times$ height $(\mathrm{cm}) 0.725] \times$ 0.007184 [11]. Patient demographic characteristics are presented as means and standard deviations for continuous variables and as proportions (percentages) for categorical variables. To test the distribution pattern, the Kolmogorov-Smirnov test was implemented. Comparisons of multiple mean values were carried out by Kruskal-Wallis tests or Analysis of Variance as appropriate. Categorical variables were compared with Chi-square test. Relationships between variables were examined by Pearson's correlation coefficient. Threshold values of the receiver operating characteristic (ROC) analysis were statistically significant. The level of significance set at $\alpha=0.05$. Statistical analyses were performed with Statistical Package for the Social Sciences (SPSS version 20.0, SPSS Inc., Chicago, IL, USA).

\section{RESULTS}

The clinical characteristics of the study groups were listed in Table 1. While there was no significant difference for gender betwen CSX group and CAD group ( $p=0.838$ ) and between CSX group and control group $(p=0.062)$, there was significant difference between control group and CAD group $(p=0.020)$. Control and CSX groups were significantly younger 
Table 2. Laboratory findings

\begin{tabular}{|c|c|c|c|}
\hline Groups & $\begin{array}{l}\text { Control } \\
(\mathbf{n}=\mathbf{8 0})\end{array}$ & $\begin{array}{c}\text { CSX } \\
(n=80)\end{array}$ & $\begin{array}{c}\text { CAD } \\
(n=80)\end{array}$ \\
\hline Glucose (mg/dL) & $92.4 \pm 3.3$ & $94.3 \pm 5.6$ & $98.2 \pm 8.1$ \\
\hline Total cholesterol (mg/dL) & $177.5 \pm 35.9$ & $183.6 \pm 49.3$ & $196.7 \pm 45.8^{\varphi}$ \\
\hline HDL-C (mg/dL) & $47.3 \pm 12.2$ & $43.1 \pm 10.3^{*}$ & $43.9 \pm 13.4^{*}$ \\
\hline LDL-C (mg/dL) & $100.5 \pm 24.2$ & $103 \pm 33.8$ & $126 \pm 37.3^{\dagger \theta}$ \\
\hline $\mathrm{TG}(\mathrm{mg} / \mathrm{dL})$ & $167.9 \pm 36.1$ & $171.6 \pm 68$ & $174.4 \pm 43$ \\
\hline WBC $\left(/ \mathrm{mm}^{3}\right)$ & $7.56 \pm 1.08$ & $8.19 \pm 1.53^{*}$ & $9.92 \pm 2.33^{\dagger \theta}$ \\
\hline Neutrophil $\left(10^{12} / \mathrm{L}\right)$ & $4.96 \pm 1.35$ & $5.87 \pm 2.17^{¥}$ & $6.18 \pm 1.28^{\dagger}$ \\
\hline Lymphocyte $\left(10^{12} / \mathrm{L}\right)$ & $2.62 \pm 0.79$ & $2.47 \pm 0.80$ & $1.89 \pm 0.63^{\dagger \ominus}$ \\
\hline NLR & $1.87 \pm 0.52$ & $2.67 \pm 0.84^{\dagger}$ & $3.63 \pm 0.38^{\dagger \theta}$ \\
\hline Erythrocyte $\left(10^{12} / \mathrm{L}\right)$ & $4.5 \pm 0.4$ & $4.7 \pm 0.8$ & $4.9 \pm 0.2$ \\
\hline Hemoglobin (g/dl) & $13.92 \pm 1.5$ & $14.0 \pm 1.81$ & $13.38 \pm 3.5$ \\
\hline MCV (fL) & $86 \pm 3.2$ & $85 \pm 2.6$ & $83 \pm 3.1$ \\
\hline Platelet & $231125 \pm 40689$ & $237500 \pm 59149$ & $235700 \pm 59251$ \\
\hline MPV & $8.03 \pm 0.65$ & $8.37 \pm 0.89^{\beta}$ & $8.69 \pm 0.88^{\dagger \bullet}$ \\
\hline $\mathrm{CRP}(\mathrm{mg} / \mathrm{dL})$ & $2.34 \pm 1.6$ & $5.41 \pm 1.39^{\dagger}$ & $7.62 \pm 2.58^{\dagger \bullet}$ \\
\hline
\end{tabular}

Data are shown as mean \pm standard deviation. $\mathrm{CAD}=$ coronary artery disease, $\mathrm{CRP}=\mathrm{C}$-reactive protein, $\mathrm{CSX}=$ cardiac syndrome $\mathrm{X}$, HDL-C = high-density lipoprotein-cholesterol, LDL-C = low-density lipoproteincholesterol, $\mathrm{MCV}=$ mean corpuscular volume, $\mathrm{MPV}=$ mean platelet volume, NLR $=$ neutrophil-lymphocyte ratio, $\mathrm{TG}=$ total triglyceride, $\mathrm{WBC}=$ white blood cell, ${ }^{*} p<0.05$ versus control group, ${ }^{\dagger} p<0.001$ versus control group, ${ }^{\beta} p<0.01$ versus control group, ${ }^{\ddagger} p<0.005$ versus control group, ${ }^{\varphi} p<0.005$ versus control group, ${ }^{\vee} p<$ 0.05 versus CSX group, ${ }^{\ominus} p<0.001$ versus CSX group

compared to the CAD group ( $p<0.0001)$, however there was no significant difference for mean age between the control group and CSX group ( $p=0.667$ ). Heart rate per minute was comparable between the CSX and control groups $(p=0.409)$, but was significantly higher in CAD group compared to the control group $(p=0.011)$ and CSX group $(p<0.001)$. There was no significant difference for BMI between the study groups. Systolic and diastolic arterial pressures were similar among all study groups. Smoking, HT and DM incidences were similar among the study groups. The hyperlipidemia incidence was higher in CAD group compared to the control and CSX groups. In the transthoracic echocardiography,
LVEF was comparable between the CSX and the control groups; but LVEF was significantly lower in CAD group as compared to the other two groups. The severity of ischaemic heart disease using Gensini score for the group with CAD was shown in Table 1.

The haematologic and biochemical parameters of the study groups were shown in Table 2 . There was no difference for glucose between the groups. Total cholesterol was similar between CAD and CSX groups; but it was significantly higher in CAD group than in control group. TG levels were similar between the study groups. LDL-C was not different between the control group and CSX group; but was significantly higher in CAD group. HDL-C was 


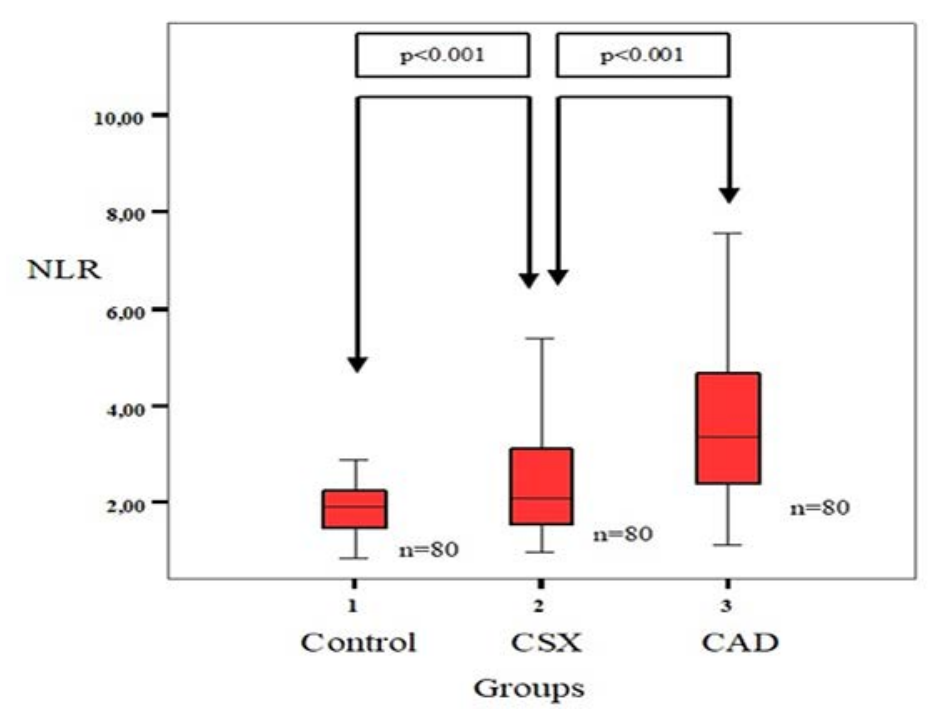

Fig. 1. Neutrophil-lymphocyte ratio of the study groups.

significantly found to be decreased both in CAD and CSX groups. There was no significant difference for haemoglobin, hematocrit and platelet counts between the study groups. Mean platelet volume (MPV) was significantly higher in CAD group compared to the other two groups; also it was higher in CSX group than in the control group. White blood cell (WBC) was higher in CAD group than CSX group $(p<0.001)$ and was higher in CSX group than control group ( $p=$ 0.022). There was no significant difference for neutrophil counts between CAD and CSX groups ( $p$ $=0.201)$; however, neutrophil count was significantly higher in CAD $(p<0.001)$ and CSX $(p=0.012)$ groups than control group. Regarding the lymphocyte counts, there was no significant difference between the control group and CSX group $(p=0.493)$, but lymphocyte count in patients with CAD group was significantly lower than CSX and the control groups. Consequently, NLR was significantly different between the study groups; NLR was higher in CAD group than CSX group and the control group that the lowest mean value was reported in the control group (Fig. 1). CRP levels were significantly different between the study groups; CRP was significantly found to be increased in CAD group and in the CSX group compared to the control group $(p<0.001)$, however it was comparable between the CAD and CSX groups $(p=0.065)$.

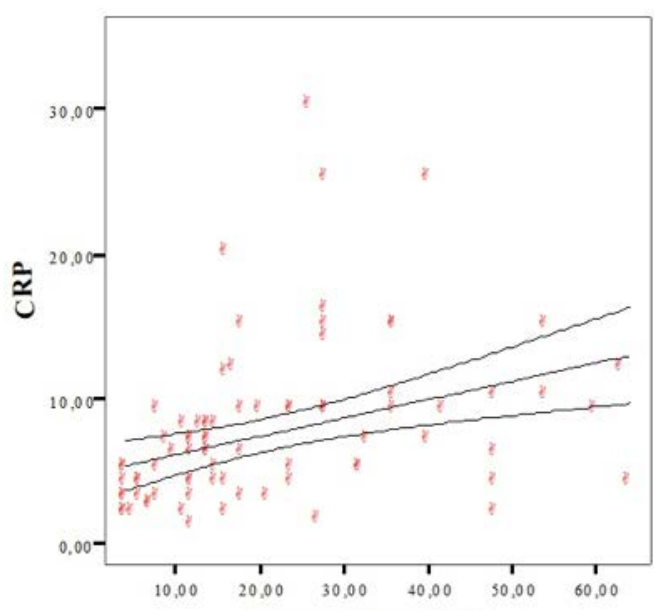

L in ear Regression with

94,50\% Me an Prediction Interval

GENSINIS CORE

Fig. 2. Correlation of CRP and Gensini score in CAD group. CAD = coronary artery disease, $C R P=C$-reactive protein 


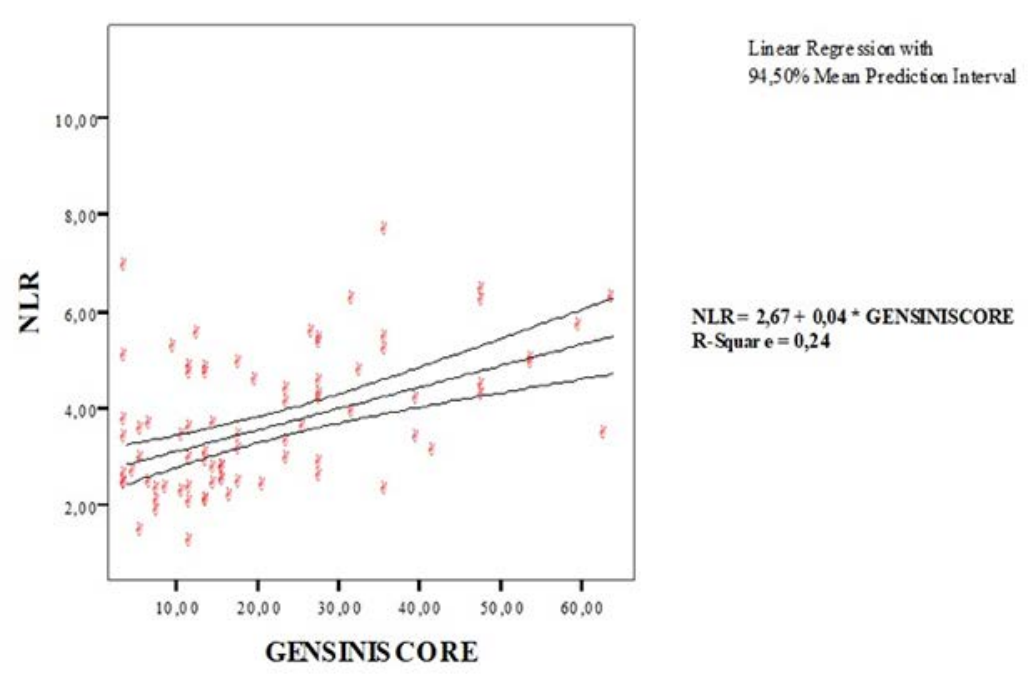

Fig. 3. Correlation of NLR and Gensini score in CAD group. CAD = coronary artery disease, NLR = neutrophil-lymphocyte ratio

In CSX, CRP was significantly positively correlated with the neutrophil count $(\mathrm{r}=0.40, p<$ 0.001 ), fairly negatively correlated with the lymphocyte count $(\mathrm{r}=-0.18, \mathrm{p}=0.077)$, significantly positively correlated with NLR $(\mathrm{r}=0.38, p=0.001)$, significantly positively correlated with WBC $(\mathrm{r}=$ $0.489, p<0.001)$. NLR was in a significant linear correlation with total cholesterol, TG and LDL-C $(\mathrm{r}=$ $0.37, p=0.001 ; \mathrm{r}=0.33, p=0.006 ; \mathrm{r}=0.38, p=$
0.001, respectively). In CAD group, CRP had significant linear correlation with WBC count $(\mathrm{r}=$ $0.33, p<0.005$ ) and was also significantly positively correlated with NLR $(\mathrm{r}=0.43, p<0.001)$ and with Gensini score ( $\mathrm{r}=0.54, p<0.001)$ (Fig. 2). In the group of CAD, NLR was also significantly correlated with Gensini score $(r=0.49, p<0.001)$ (Fig. 3).

The ROC curve analysis was performed to compare the abilities of CRP, WBC and NLR in

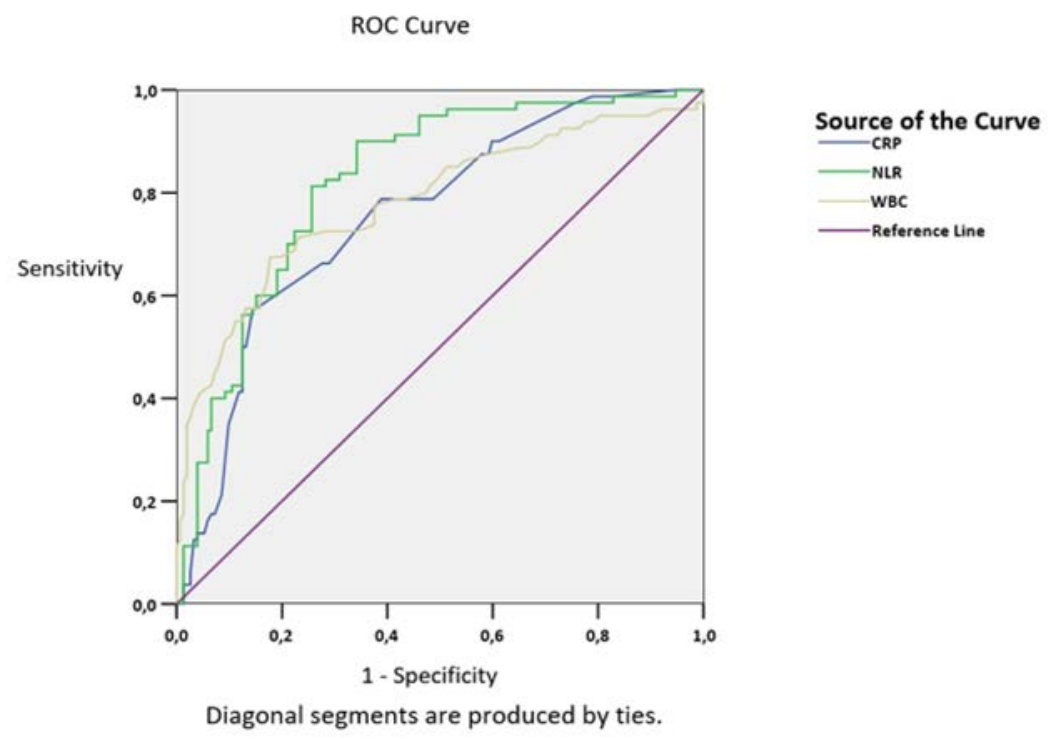

Fig. 4. ROC curve analysis for the ability of NLR, CRP and WBC to predict obstructive CAD in patients with chest pain. For NLR; the area under the curve (AUC) is 0.823 (95\% confidence interval [CI]: $0.768-0.878)$. NLR = 2.26; the sensitivity was $85.5 \%$ and the specificity was $43.9 .6 \%$. For WBC, the AUC is $0.781(95 \%$ CI: $0.714-0.849)$. WBC $=9.5(\times 109 / \mathrm{L})$; the sensitivity was $58.8 \%$ and the specificity was $34.3 \%$. For CRP, the AUC is 0.756 (95\% CI: $0.692-0.820)$. CRP $=3.05 \mathrm{mg} / \mathrm{dL}$; the sensitivity was $78.7 \%$ and the specificity was $43.6 \%$. CAD $=$ coronary artery disease, $C R P=C$-reactive protein, $N L R=$ neutrophil-lymphocyte ratio, $\mathrm{ROC}=$ receiver operating characteristic, $\mathrm{WBC}=$ white blood cell 
predicting obstructive coronary artery disease including coronary artery narrowing $>50 \%$ of the coronary vessel diameter in patients with typical chest pain. We examined the sensitivity and specificity of CRP, WBC and NLR for detection of coronary artery disease at different CRP, WBC and NLR values; whereas NLR provided excellent sensitivity and specificity for the detection of obstructive coronary artery disease. In patients of the three groups, when the cut-off value was taken as 2.26 for NLR, NLR values attained a $85.5 \%$ sensitivity and $43.9 \%$ specificity $(p<0.001)$. When the cut-off value was taken as $3.05 \mathrm{mg} / \mathrm{dL}$ for CRP, CRP values attained a $78.7 \%$ sensitivity and $43.6 \%$ specificity $(p<0.001)$. When the cut-off value was taken as $9.5(\times 109 / \mathrm{L})$ for WBC, WBC values attained a $58.8 \%$ sensitivity and $34.3 \%$ specificity $(p<0.001)$ (Fig. 4$)$. It seems that NLR significantly predicted obstructive coronary artery disease in patients with chest pain.

\section{DISCUSSION}

The main finding of our study was that, NLR, CRP and WBC were significantly increased in patients with obstructive CAD compared to the patients with CSX and control group. Additionally, in patients with CSX, NLR, CRP and WBC were significantly increased compared to the control group. NLR was found significant in determining obstructive coronary artery disease in patients with typical chest pain and coronary risk factors.

The role of inflammation in CAD has been shown in several studies [12]. CRP, that's the prototype of acute phase proteins, is a marker of systemic inflammation, elevated in response to injury, infection, and various inflammatory stimuli [13]. CRP is related to some cardiovascular risk factors, such as obesity, smoking, increased blood pressure, serum lipids, increased blood glucose, and inversely to HDL-C levels $[14,15]$. CRP was also reported as an important biomarker in predicting obstructive CAD [16]. In the current study, CRP was significantly increased in patients with CSX and obstructive CAD as compared to the control group; there was no significant difference for CRP between patients with obstructive CAD and patients with CSX. This finding implicated that CSX has an inflammatory underlying pathology.
In several previous studies, systemic inflammation was found related with microvascular endothelial dysfunction which has been proposed as the main pathophysiological mechanism of CSX [17]. Inflammation that can be assessed with CRP, has a significant detrimental effect during the impairment of endothelium-dependent vasodilator function in patients with CSX [18]. Teragawa et al. [19] investigated the relation between CRP concentrations and coronary microvascular endothelial function in patients with chest pain and angiographically normal coronary arteries. They found inverse and independent correlation between CRP levels and acetylcholineinduced changes in coronary blood flow. In the study of Tousoulis et al. [20] the researchers reported increased blood levels of vascular cell adhesion molecule-1 (VCAM-1) and intercellular adhesion molecule-1 (ICAM-1), adhesion molecules that are synthesized by activated endothelial cells in response to inflammatory stimuli, in patients with cardiac syndrome $\mathrm{X}$.

NLR reflects the balance between neutrophil and lymphocyte levels in peripheral blood and recently has been shown as an indicator of systemic inflammation [21]. In various studies, higher NLR value has been reported be associated with adverse clinical outcomes in patients with CAD. During the hospitalization, NLR was an independent predictor of in-hospital and 6month mortality in patients with ACS. NLR was also an independent predictor of 1- and 6-month and 4-year mortalities in patients with non-STEMI and STelevated myocardial infarction (STEMI) $[22,23]$. In our observational study, NLR was significantly increased in patients with obstructive CAD when compared to patients with CSX and control group. Furthermore, NLR was significantly increased in patients with CSX compared to the control group. This result stated that, beside the CRP, NLR was also useful in prediction of obstructive CAD in patients with typical chest pain. Additionaly, this simple readily available marker may provide a risk stratification in patients with typical anginal chest pain beyond the conventional risk scores.

\section{Limitations}

Our study has some limitations which should be mentioned. First, the study population was relatively small. Second, an intravascular imaging was not 
carried out at baseline assessment. Therefore, it was unknown whether any of the patients with CSX had atherosclerosis not identifiable by coronary angiography. Third, we did not perform measurements related with endothelial dysfunction in patients with CSX. Fourth, we could not measure levels of inflammatory proteins (i.e., TNFalpha, IL-6) because of lack of technical facilities in our center.

\section{CONCLUSION}

NLR is usefull in prediction of obstructive coronary artery disease in patients with typical anginal chest pain and conventional risk factors. In patients with CSX, increased CRP, WBC and NLR may give insights about the main pathology of this disease. Despite the absence of evident obstructive narrowing in epicardial coronaries, it seems salutary to treat CSX, since it has the same underlying pathophysiology with obstructive CAD.

\section{Conflict of interest}

The authors disclosed no conflict of interest during the preparation or publication of this manuscript.

\section{Financing}

The authors disclosed that they did not receive any grant during conduction or writing of this study.

\section{REFERENCES}

1. Hoffman M, Blum A, Baruch R, Kaplan E, Benjamin M. Leukocytes and coronary heart disease. Atherosclerosis 2004; 172:1-6.

2. Gillum RF, Mussolino ME, Madans JH. Counts of neutrophils, lymphocytes, and monocytes, cause-specific mortality and coronary heart disease: the NHANES-I epidemiologic follow-up study. Annals of epidemiology 2005; 15:266-71.

3. Libby P. Vascular biology of atherosclerosis: overview and state of the art. Am J Cardiol 2003;91(3A):3A-6A.

4. Nunez J, Nunez E, Bodi V, Sanchis J, Minana G, Mainar L, et al. Usefulness of the neutrophil to lymphocyte ratio in predicting long-term mortality in ST segment elevation myocardial infarction. Am J Cardiol 2008;101:747-52.

5. Horne BD, Anderson JL, John JM, Weaver A, Bair TL, Jensen $\mathrm{KR}$, et al. Which white blood cell subtypes predict increased cardiovascular risk? J Am Coll Cardiol 2005;45:1638-43.

6. Walsh SR, Cook EJ, Goulder F, Justin TA, Keeling NJ.
Neutrophil-lymphocyte ratio as a prognostic factor in colorectal cancer. J Surg Oncol 2005;91:181-4.

7. Duffy BK, Gurm HS, Rajagopal V, Gupta R, Ellis SG, Bhatt DL. Usefulness of an elevated neutrophil to lymphocyte ratio in predicting long-term mortality after percutaneous coronary intervention. Am J Cardiol 2006;97:993-6.

8. Dentali F, Nigro O, Squizzato A, Gianni M, Zuretti F, Grandi $\mathrm{AM}$, et al. Impact of neutrophils to lymphocytes ratio on major clinical outcomes in patients with acute coronary syndromes: A systematic review and meta-analysis of the literature. Int $\mathrm{J}$ Cardiol 2018;266:31-7.

9. Kaski JC, Rosano GM, Collins P, Nihoyannopoulos P, Maseri A, Poole-Wilson PA. Cardiac syndrome X: clinical characteristics and left ventricular function. Long-term follow-up study. J Am Coll Cardiol 1995;25:807-14.

10. Al Suwaidi J, Higano ST, Holmes DR Jr, Lerman A. Pathophysiology, diagnosis, and current management strategies for chest pain in patients with normal findings on angiography. Mayo Clin Proc 2001;76:813-22.

11. Bland JM, Altman DG. Statistical methods for assessing agreement between two methods of clinical measurement. Lancet 1986;1:307-10.

12. Libby P. Changing concepts of atherogenesis. J Intern Med 2000;247:349-58.

13. Deodhar SD. C-reactive protein: the best laboratory indicator available for monitoring disease activity. Cleve Clin J Med 1989;56:126-30.

14. Cook DG, Mendall MA, Whincup PH, Carey IM, Ballam L, Morris JE, et al. C-reactive protein concentration in children: relationship to adiposity and other cardiovascular risk factors. Atherosclerosis 2000;149:139-50.

15. Mendall MA, Patel P, Ballam L, Strachan D, Northfield TC. $\mathrm{C}$ reactive protein and its relation to cardiovascular risk factors: a population based cross sectional study. BMJ 1996;312:1061-5. 16. Sharma S, Ghalaut VS, Dixit R, Kumar S, George PJ. Microalbuminuria and C-reactive protein as a predictor of coronary artery disease in patients of acute chest pain. J Cardiovasc Dis Res 2013;4:37-9.

17. Lekakis JP, Papamichael CM, Vemmos CN, Voutsas AA, Stamatelopoulos SF, Moulopoulos SD. Peripheral vascular endothelial dysfunction in patients with angina pectoris and normal coronary arteriograms. J Am Coll Cardiol 1998;31:5416.

18. Tondi P, Santoliquido A, Di Giorgio A, Sestito A, Sgueglia GA, Flore R, et al. Endothelial dysfunction as assessed by flowmediated dilation in patients with cardiac syndrome $\mathrm{X}$ : role of inflammation. Eur Rev Med Pharmacol Sci 2011;15:1074-7.

19. Teragawa H, Fukuda Y, Matsuda K, Ueda K, Higashi Y, Oshima $\mathrm{T}$, et al. Relation between $\mathrm{C}$ reactive protein concentrations and coronary microvascular endothelial function. Heart 2004;90:750-4.

20. Tousoulis D, Davies GJ, Asimakopoulos G, Homaei H, Zouridakis E, Ahmed N, et al. Vascular cell adhesion molecule1 and intercellular adhesion molecule- 1 serum level in patients with chest pain and normal coronary arteries (syndrome X). Clin Cardiol 2001;24:301-4.

21. Kalay N, Dogdu O, Koc F, Yarlioglues M, Ardic I, Akpek M, 
et al. Hematologic parameters and angiographic progression of coronary atherosclerosis. Angiology 2012;63:213-7.

22. Tamhane UU, Aneja S, Montgomery D, Rogers EK, Eagle KA, Gurm HS. Association between admission neutrophil to lymphocyte ratio and outcomes in patients with acute coronary syndrome. Am J Cardiol 2008;102:653-7.

23. Azab B, Zaher M, Weiserbs KF, Torbey E, Lacossiere K, Gaddam S, et al. Usefulness of neutrophil to lymphocyte ratio in 